\title{
Análisis de comportamiento de articulación en un manipulador de tres grados de libertad
}

Diana J. Lancheros C.

Recibido el 22 de Febrero de 2011 - Aprobado el 24 de Mayo de 2011

\section{Resumen}

El desarrollo de modelos matemáticos para la simulación y el análisis de comportamientos en sistemas mecánicos, eléctricos, hidráulicos y neumáticos, permiten determinar sistemas de control óptimos. El presente artículo muestra el análisis de comportamiento de una articulación de un manipulador, que actualmente se utiliza en el laboratorio de electrónica de la Corporación Universitaria Minuto de Dios (UNIMINUTO). La simulación realizada del sistema, permitió identificar la variación de la posición de los eslabones en función del voltaje aplicado sobre el motor que mueve la articulación.

\section{Palabras clave}

Modelación matemática, grados de libertad, función de transferencia, simulación.

\begin{abstract}
The development of mathematical models for simulation and analysis of behavior in mechanical, electrical, hydraulic and pneumatic systems for determining optimal control. This article shows the analysis of behavior of a joint of a manipulator, which is currently, used in the electronics laboratory Corporación Universitaria Minuto de Dios (UNIMINUTO). System simulation performed, identifying a change in the position of the links based on the voltage applied to the motor that moves the joint.
\end{abstract}

\section{Keywords}

Mathematical models, degrees of freedom, transfer function, simulation.

\section{Introducción}

Actualmente, en el laboratorio de electrónica del Programa de Tecnología en Electrónica de UNIMINUTO, se realizan prácticas con un manipulador robótico, a fin de dar a los estudiantes instrumentos que simulen a escala contextos reales. Las prácticas de mayor frecuencia se realizan en el diseño y la programación de sistemas de producción, actividades con trabajos repetitivos para el ensamble y manipulación de objetos. Para el 
desarrollo óptimo de dichos ejercicios, se hizo necesario el análisis del comportamiento de la parte eléctrica y mecánica sobre el eslabón que presenta mayor inconveniente. A continuación, se muestra en primera instancia una revisión de trabajos relacionados, donde herramientas pedagógicas como robots permiten mejorar el aprendizaje y el desarrollo de las prácticas de laboratorio. Luego, se realiza el análisis matemático de los componentes electrónicos que controlan una articulación del robot, hasta obtener la función de transferencia que la representa. Se procede luego a realizar la simulación en Matlab ${ }^{\circledR}$ para analizar el comportamiento, y por último se hacen mediciones prácticas para comparar valores y determinar las características de comportamiento.

\section{Antecedentes}

Realizando una revisión de los sistemas, módulos y bancos didácticos existentes para la enseñanza de la robótica se encuentran propuestas interesantes como los Legos Mindstorms; al respecto Dylan Evans (2004) realizó una investigación experimental en donde afirmó que se puede utilizar una herramienta de aprendizaje para hacer más productivo el conocimiento de la robótica en estudiantes de primer año de ingeniería. En el proceso de experimentación, el cual durante todo el curso utilizó Legos Mindstorms ${ }^{\circledR}$; los resultados encontrados incluyeron: mayor receptividad de los estudiantes al no tener un curso teórico de un nivel de dificultad alto, mayor análisis en los problemas de diseño de sistemas robóticos planteados utilizando el Lego, cumplimiento de todos los objetivos del curso sin dejar atrás ningún tema de los planteados.

El principal aporte que se puede tomar de esta investigación está enfocado al uso de los Legos como herramienta pedagógica en la cual existe una interacción del diseño físico y el software. Los estudiantes descubren que muchos de los problemas de la robótica pueden ser solucionados de forma sencilla, el tiempo que se invierte en la construcción mecánica de los robots es aprovechado en el diseño avanzado de sistemas electrónicos y algoritmos de programación.

Moundridou y Kalinoglou (2008), realizaron una investigación en términos de eficacia en la utilización de robots Legos en las aulas de clase, los resultados del estudio fueron positivos en términos del alto nivel de aprendizaje obtenido por los estudiantes y los cortos tiempos de desarrollo e implementación de prácticas de laboratorio con robots móviles. El enfoque dado en el curso fue útil para ayudar a comprender los conceptos que los estudiantes tenían en torno a la temática. El trabajo presenta niveles efectivos de motivación en aulas de clase de robótica, donde el aporte de la matemática es fuerte y muchas veces dificulta el proceso de aprendizaje.

En el mismo año, Grega y Pilat (2008) realizaron un estudio comparativo de diferentes herramientas de programación que pueden ser utilizadas con robots Legos Mindstorms ${ }^{\circledR}$, evidenciando la flexibilidad de la herramienta didáctica y la gran utilidad que presta cuando se utiliza en clases de robótica.

En el año 2005, O'Hara \& Ka (2005) realizaron una investigación acerca de la utilización del software libre en la robótica educativa, y su principal aporte en el aprendizaje, la enseñanza, la utilización, y el desarrollo de robots y sus correspondientes aplicaciones tecnológicas. Esta investigación se basó en dos casos de estudio, el primero realizó un programa hecho en plataforma Linux (open source) para el posicionamiento de un robot móvil por medio del mouse, en este proyecto los resultados fueron óptimos en cuanto a eficiencia, rapidez y funcionamiento. En el segundo caso de estudio, los resultados de la realización del software que manejó lenguaje natural para la programación del Lego, generó resultados positivos en cuanto a que el sistema desarrollado fue más entendible que los sistemas propios del kit Lego. La conclusión más importante al respecto, afirma que el software libre ha demostrado ser fiable y potente para las soluciones de proyectos educativos en los cuales intervienen diseños con robots.

Durante el mismo año. Illah Nourbakhsh, y Hammer (2005) implementaron un sistema basado en medidas formales de aprendizaje en un curso de robótica móvil en conjunto con la NASA, la metodología utilizada se dividió en etapas especificas: en primera instancia se pidió a los estudiantes la construcción mecánica del robot, luego se diseñó el lenguaje de programación, se acopló el sistema electrónico del robot, se hizo un análisis del nivel de aprendizaje de los estudiantes en cada una de las etapas, se tabularon los resultados y se emitieron conclusiones. Durante las primeras cuatro semanas los estudiantes presentaron un nivel de satisfacción de 4 en una escala de 1 a 5, pero al final para la entrega, por la presión, los estudiantes no se sintieron conformes. El grupo de estudiantes que realizaron proyectos de robots para los concursos presentaron mayor satisfacción. Las actividades de mayor participación estuvieron determinadas por temas de solución de problemas, trabajo en equipo e identificación con la tecnología. Esto sugiere que en un curso de robótica para cumplir los objetivos de desarrollo y dominar el interés general, se puede preparar a los estudiantes con habilidades 
para el éxito en los proyectos de desarrollo que involucran ciencia y educación.

Por su parte, Lin, Zhi-Feng Liu, Chan-Hsin, y Virnes (2009) realizaron una investigación para entender el efecto de la robótica Lego ${ }^{\circledR}$ utilizada en un curso. Los resultados evidenciaron creatividad en los estudiantes y desarrollo de habilidades en la resolución de problemas. Durante este proceso, se utilizaron tres estrategias, en la primera se colocaban ejercicios con dificultad leve, en la segunda se asignaban nuevos elementos al problema anterior, y en la tercera se redefinían los modelos de los robots a partir del primer problema planteado. Después de analizar el proceso de resolución creativa de ejercicios, el resultado mostró que la información detallada que presentaba cada una de las actividades era favorable para el entendimiento y resolución de problemas en el momento de ensamblar un modelo de robot móvil. De forma adicional, el estudiante desarrolló habilidades de pensamiento creativo y de motivación en el desarrollo de una tarea.

Este trabajo aporta significativamente a la investigación, ya que evidencia el desarrollo de capacidades como la creatividad, el trabajo en equipo, el diseño del robot, y la resolución de problemas.

Así mismo, Mosley y Doswell (2008) realizaron una investigación en donde evidenciaron la importancia de utilizar robots Lego ${ }^{\circledR}$ para el desarrollo de habilidades matemáticas en estudiantes de un curso de robótica. La estrategia utilizada consistió, en primera instancia, en presentar gráficas de cada una de las piezas y explicar el funcionamiento, seguido de la asignación de problemas específicos que consistían en la construcción de modelos con las fichas explicadas. De los resultados arrojados en estas etapas, se diseñó e implementó un instructor virtual cuyo objetivo era brindar de forma secuencial, la forma como debían construirse cada uno de los modelos. El instructor virtual proporcionó un aprendizaje personalizado mediante la aplicación de la experiencia humana y técnicas de instrucción que también permitieron mejorar el aprendizaje en los estudiantes del curso de robótica móvil.

El principal aporte de esta investigación obedece a que existen tres conjuntos de habilidades que los estudiantes necesitan para adquirir los conocimientos necesarios a fin de manipular óptimamente un robot, la primera el desarrollo de la habilidad espacial para el diseño y la construcción, el segundo grupo está conformado por el desarrollo de habilidades matemáticas y por último el desarrollo de competencias básicas de programación. El éxito de la construcción de un robot móvil implica que el estudiante no sólo sea capaz de reconocer una pieza de Lego $^{\circledR}$, sino que interprete e identifique de manera adecuada su funcionamiento. A su vez, Hirst, Johnson, Petre, Price, y Richards (2003) encontraron en su investigación que los métodos tradicionales de enseñanza de la informática tienden a ser abstractos, y los estudiantes suelen presentar dificultades en el razonamiento lógico matemático. Afirman que hay varias ventajas en la enseñanza de la implementación y programación de robots con entornos adaptativos que proporcionen fuertes estímulos visuales y apoyen el correcto desarrollo de la habilidad espacial. Esta estrategia permite de forma adicional desarrollar en los estudiantes la creatividad, la manipulación del contexto espacial, la motivación en el planteamiento de nuevas problemáticas a partir de los problemas planteados inicialmente. Este trabajo evidencia la necesidad de generar entornos gráficos para el desarrollo de investigaciones espaciales en un curso de robótica.

\section{Planteamiento del problema}

El planteamiento del problema se enfoca en la realización del análisis matemático, modelamiento y simulación del eslabón superior de un robot de tres grados de libertad, teniendo en cuenta la posición del eje y el voltaje de alimentación del motor.

A continuación, se presenta la fotografía del autómata sobre el cual se pretende realizar el modelamiento matemático. Además, se especifica el eslabón sobre el cual se realizó el análisis.

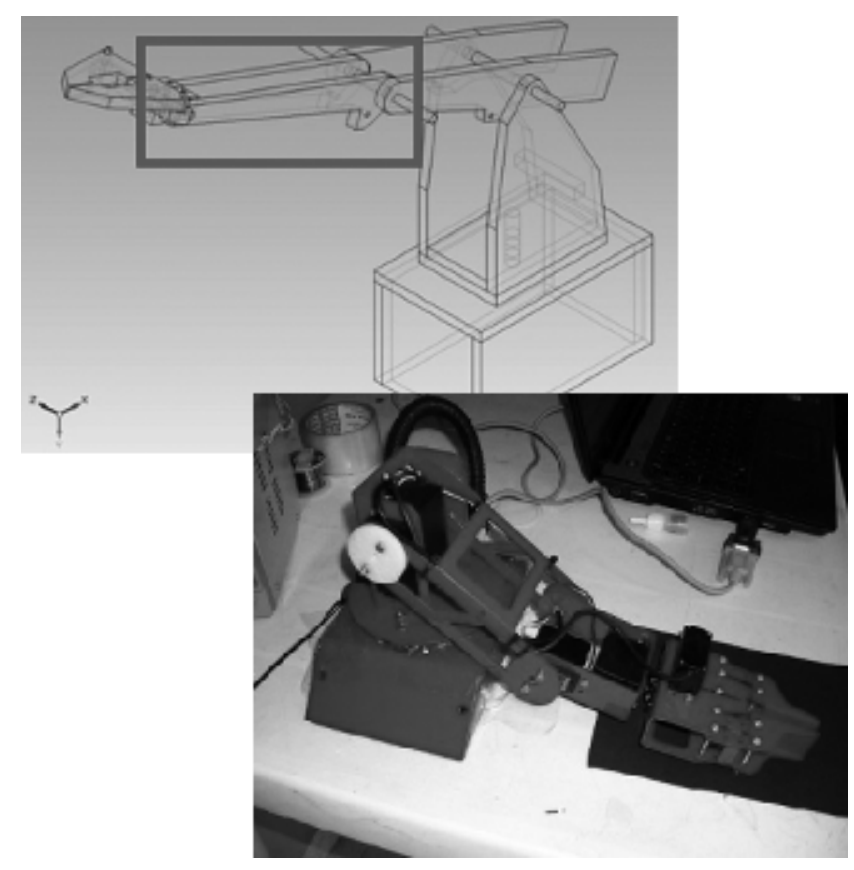

Figura 1. (a). Eslabón de análisis. (b) Construcción Manipulador. 2010 


\section{Metodología}

En primer lugar, es necesario conocer el esquema del sistema sobre el cual se pretende realizar el modelo matemático y la simulación. Dicho sistema se muestra en la figura 2.

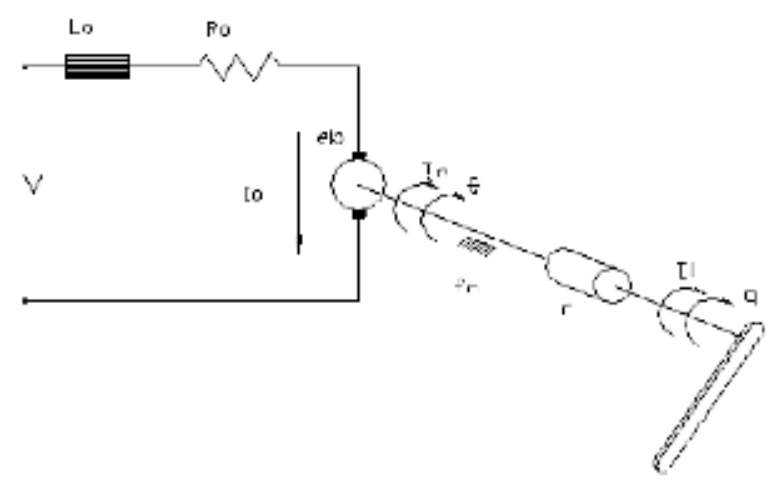

Figura 2. Esquema del sistema eléctrico-mecánico del brazo superior del manipulador de tres grados de libertad. (Kuo, 2000)

Una vez determinado el esquema del sistema, es necesario conocer los valores de los elementos que lo componen tanto eléctricos como mecánicos para su posterior modelamiento y simulación como se ilustra a continuación.

\section{Valores conocidos:}

Los valores conocidos se muestran a continuación:

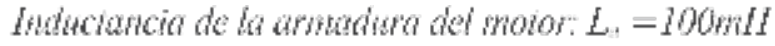
lolaje de abmentaton du motor: $V_{n}=9 \mathrm{~W}$

Resistentia de ia armadura del molor: $R_{i}=23 \Omega$ Velchidad angeslar del molor: $(\omega)=152 \mathrm{rpm}$

Man del notor (nasa del punfol: $n=4 \mathrm{Jw}=0.04 \mathrm{~kg}$. Distancia al gie de rotation: $r=0.1013 \mathrm{~m}$

\section{Valores obtenidos:}

Par motor, la ecuación (1) muestra la ecuación para calcular el valor:

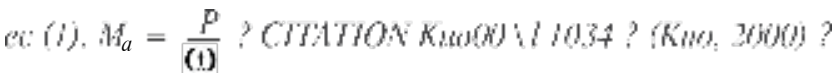

Para lo cual es necesario conocer el valor de P (potencia) teniendo en cuenta la corriente, usando la ecuación (2)

$$
\text { ec (2), } P=\mathrm{V}_{i} \mathrm{I}_{(\mathrm{Kuo}, 2000)}
$$

Por su parte, I se determina con el voltaje de alimentación del motor y la resistencia de la armadura del motor, utilizando la ecuación (3)

$$
\text { ec (2). } I=\frac{V_{i}}{R}(\text { Kuo, 2000) }
$$

$I=\frac{9 V}{23 \Omega}$

$I=0.39 \mathrm{~A}=390 \mathrm{~mm}$

Entonces, aplicando la ecuación (2), la potencia del motor será:

$P=9 V * 0.39 \mathrm{~A}$

$P=3.52 \mathrm{~W}$

Y finalmente, el par motor necesario para el sistema es calculado con ecuación (1):

$M_{o}=\frac{3.52 \mathrm{~W}}{152 \mathrm{mpm}}$

$M_{C}=\frac{3.52 \mathrm{~W}}{15,9 \mathrm{rad}}$

$M_{\alpha}=0.221 \mathrm{Mm}$

El momento de inercia del motor y el reductor se calcula teniendo en cuenta la ecuación (4):

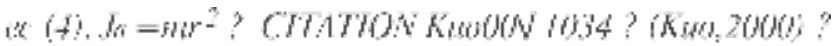

$J_{\mathrm{i}}=0.0 \mathrm{~s} / \mathrm{Ku} \cdot{ }^{*}(0.0 \mathrm{~s} \cdot 3 \mathrm{~m})^{2}$

$f_{\mathrm{i}}=0.0006989 \mathrm{Kg}: \mathrm{mi}^{2}$

1. Para realizar el modelo matemático del sistema y obtener la función de transferencia del eslabón deseado, es necesario partir del circuito de un motor de corriente continua al cual se le aplica una carga. Aplicando leyes de Kirchhoff al circuito eléctrico de la figura 1(a), se obtiene la ecuación (5):

$$
e c(5) . \quad V(j)=R_{a} I_{\alpha}+L \frac{d L_{j}}{d i}+\epsilon_{i} \quad(\mathrm{Kuo}, 2000)
$$

Donde $\ell_{\nu}$ es la fuerza contraelectromotriz del motor. Es necesario por tanto, que se calcule teniendo en cuenta ecuación (6):

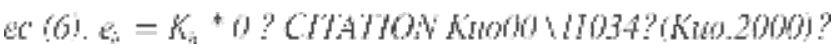

En donde $K$ es la constante de fuerza contraelectromotriz del motor y $\theta$ es la posición angular del eje del motor. Posteriormente, se aplica la transformada de Laplace a la ecuación obtenida anteriormente, y se despeja la corriente de la misma como se observa en las ecuaciones (7) y (8). 


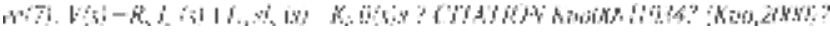

$$
\text { e(s). } f_{n}(s)=\frac{V(s)-h_{s} O(s) s}{R_{u}+s L_{s}}(\text { Kuo, 2000) }
$$

Teniendo en cuenta que el par desarrollado por el motor es igual a la suma de los pares disipados por el motor y su carga reflejada en el eje del motor, se tiene la ecuación (9).

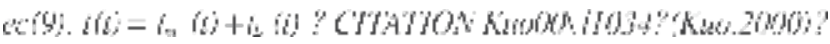

Entonces, los pares del motor y la carga se obtienen teniendo en cuenta las ecuaciones (10) y (11).

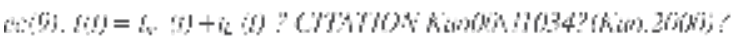

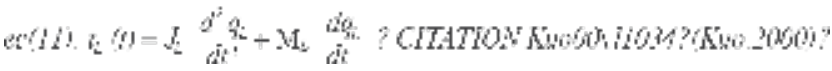

Posteriormente, es importante analizar que el trabajo realizado por la carga respecto a su eje es igual al trabajo realizado por la carga referida al eje del motor, esto se relaciona teniendo en cuenta las ecuaciones (12), (13), (14), (15 (16).

$$
\begin{aligned}
& \text { ectlis } \left.t_{s}(t)=\frac{T_{r}(t)}{r} \quad(k, 0), 2000\right)
\end{aligned}
$$

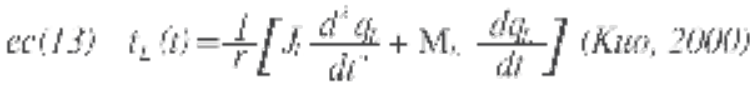

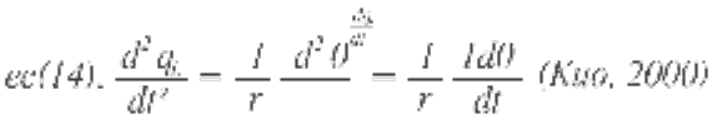

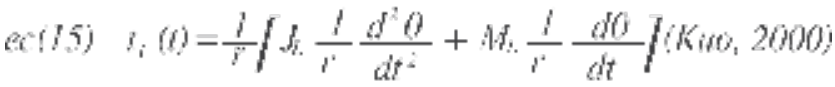

$$
\begin{aligned}
& \text { ec(16) } i_{L}(t)=\frac{l}{d}\left[J \frac{d^{2} \theta}{d t^{2}}+M \frac{d \theta}{d t}\right] \text { (Kuo. 2000) }
\end{aligned}
$$

Reemplazando en la ecuación (12), las expuestas para la carga y aplicando la transformada de Laplace se obtienen las ecuaciones (17) y (18).

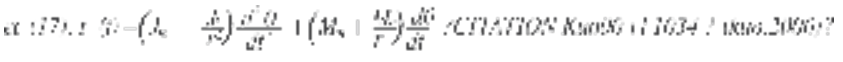

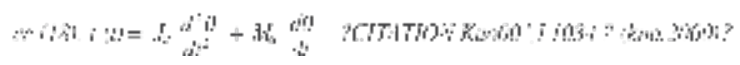

Aplicando la transformada de Laplace a la ecuación (18), se tiene entonces la ecuación (19) que calcula el torque:

$\operatorname{ec}(19) . T(s)=s^{2} J_{n} 0(s)+M_{0} 0(5)(k w 0.2000)$

Se debe tener en cuenta que el torque en el motor es directamente proporcional a la corriente que circula por su armadura y teniendo en cuenta la ecuación (19) obtenida para la corriente en el dominio de la frecuencia, se llega a la ecuación (20): $e(20) \cdot T(s)=K_{n}\left[\frac{V(s)-K_{u} 0(s) s}{R_{a}+L_{s, z} s}\right]\left(k_{u 0}, 2000\right)$

Esto debido a que la relación del torque en el motor para el movimiento del brazo superior del manipulador es el producto entre la constante del par del motor y la corriente necesaria para la activación del mismo. Finalmente, para obtener la función de transferencia del sistema en función de la posición del brazo y el voltaje necesario, se igualan las ecuaciones obtenidas para T(s), ecuación (19), como se muestra a continuación el procedimiento dado las ecuaciones (21), (22), (23), (24).

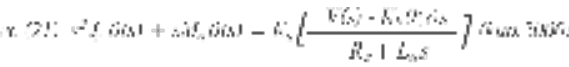

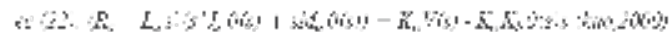

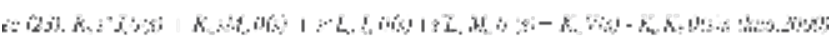

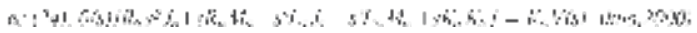

Finalmente, la función de transferencia del sistema es la que se muestra en la ecuación (25).

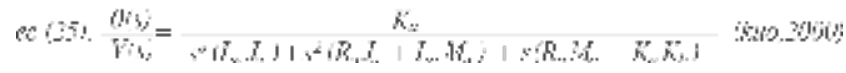

Antes de realizar la simulación en MatLAB ${ }^{\circledR}$ y Simulink ${ }^{\circledR}$ es necesario conocer el valor de $\mathrm{Kb}$ el cual se obtiene teniendo en cuenta las ecuaciones de potencia, par en el eje del motor y fuerza contraelectromotriz del motor, esto se evidencia en las ecuaciones (26), (27), (28) y (29).

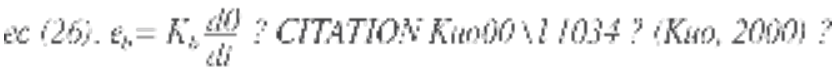

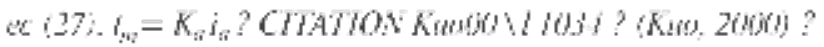

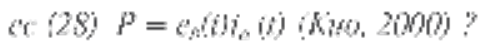

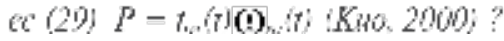

Reemplazando las ecuaciones (26) y (27) en las ecuaciones (28) y (29) se obtienen las ecuaciones (30) y (31).

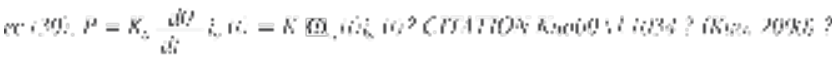

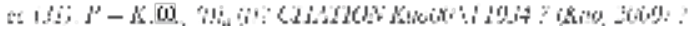

Igualando las dos ecuaciones anteriores se llega entonces a la siguiente conclusión:

$$
K_{i s}=K_{i}
$$

Para finalizar, es necesario hallar el valor de $K_{a}$ y $K_{a}$ por medio de las ecuaciones (32) y (33).

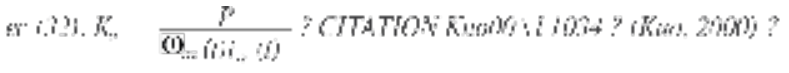

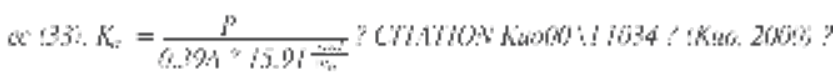

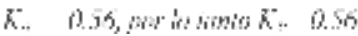


2. Una vez se obtienen los parámetros se realiza la simulación del sistema, en Simulink ${ }^{\circledR}$ mediante el diagrama de bloques, como se muestra en la figura 3.

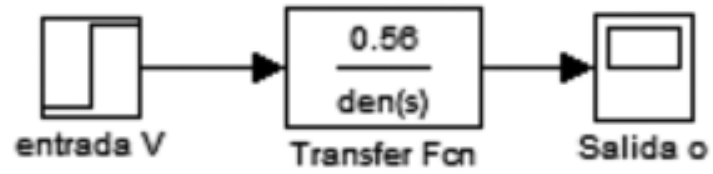

Figura 3. Diagrama de bloques de la función de transferencia 2010

La figura 4 muestra el ingreso de parámetros en el sistema.

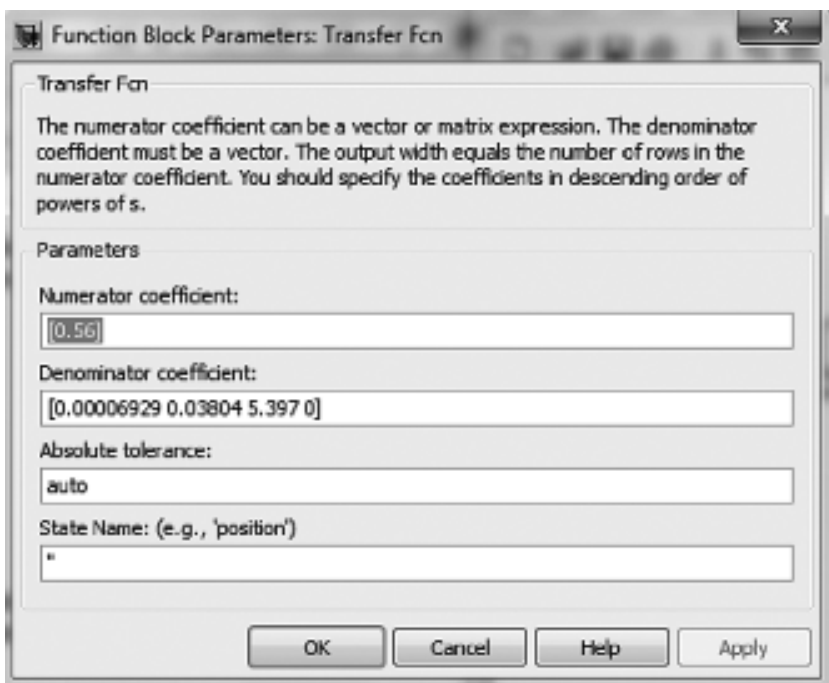

Figura 4. Parámetros de la función de transferencia del bloque Transfer Fcn, 2010.

Una vez realizado el modelo en Simulink ${ }^{\circledR}$ se reproduce para obtener la simulación de la figura 5.

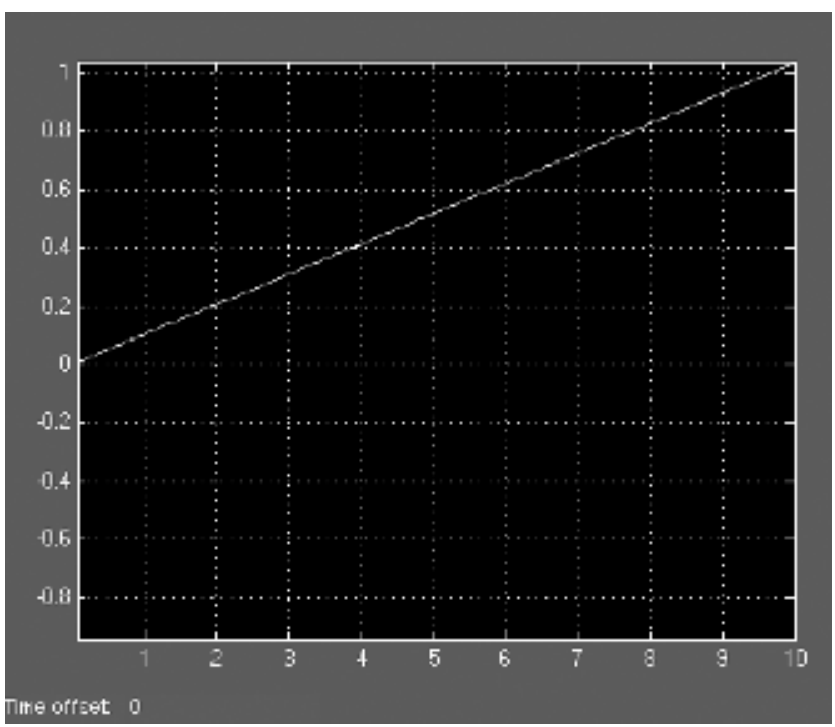

Figura 5. Gráfica de la simulación obtenida en Simulink ${ }^{\circledR} 2010$

En la figura anterior se aprecia, que a una entrada de voltaje determinada, el sistema responderá modifi- cando la posición constantemente, algo que resulta indiscutible, puesto que la entrada de voltaje en el motor provocará un movimiento continuo del eslabón del autómata.

3.Construcción del modelo en MatLAB ${ }^{\circledR}$ y simulación. El código empleado para la construcción de la función de transferencia y su posterior simulación se muestra a continuación.

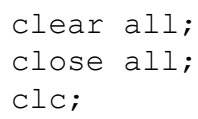

oConstantes de la función de transferencia

oConstante de par del motor, resistencia, inductancia,

oMomento de inercia del motor, par del motor $\mathrm{Ka}=0.56$;

$\mathrm{Kb}=0.56$;

$\mathrm{Ra}=23$;

$\mathrm{La}=0.1 ;$

$\mathrm{Ja}=0.0006929$;

$\mathrm{Ma}=0.221$;

num $=[\mathrm{Ka}]$

$\mathrm{den}=\left[\begin{array}{lll}(\mathrm{Ja} * \mathrm{La}) & (\mathrm{Ja} * \mathrm{Ra}+\mathrm{La} * \mathrm{Ma}) \quad(\mathrm{Ra} * \mathrm{Ma}+\mathrm{Ka} * \mathrm{~Kb}) \quad 0\end{array}\right]$

$\mathrm{Gs}=\mathrm{tf}$ (num, den)

step (Gs)

grid on

$\operatorname{axis}\left(\left[\begin{array}{llll}0 & 10 & 0 & 1\end{array}\right]\right)$

title('Respuesta al escalón')

Claramente se denota que en un principio, es importante declarar las variables necesarias para la construcción de la función de transferencia, una vez realizado esto, se define la función de transferencia del sistema, y finalmente se determina la respuesta al escalón del sistema, el cual se muestra en la figura 6.

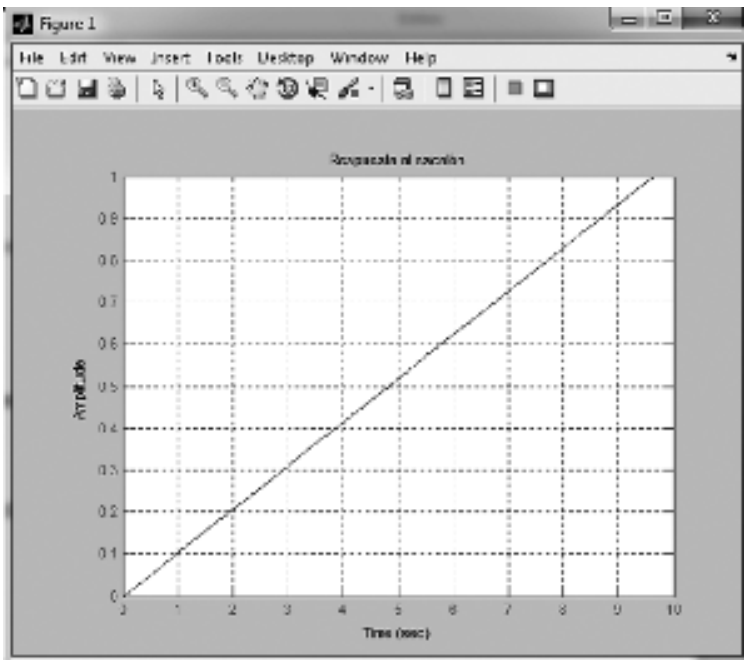

Figura 6. Respuesta al escalón del sistema, realizado en Mat$L A B^{\circledR} 2010$ 
Al igual que en la simulación realizada en Simulink ${ }^{\circledR}$, el crecimiento de la posición en el tiempo en respuesta al voltaje es continuo, lo que permite conocer el comportamiento del eslabón del robot cuando se aplica un voltaje determinado en el motor del eje del eslabón.

4. Mediciones experimentales del comportamiento Para complementar el análisis del comportamiento del eslabón, es necesario realizar la experimentación, en donde se mide el cambio de posición del autómata variando el voltaje desde 0 hasta $9 \mathrm{~V}$, que es la capacidad máxima de los motores. A continuación se muestran los resultados obtenidos para el sistema.

\begin{tabular}{|c|c|c|c|c|c|}
\hline Posicion(grados) & Tiempo(seg) & Voltaje(V) & Grados una vuelta & tiempo una vuelta & Posicion angular (rpm) \\
\hline 70 & 0 & 0 & 360 & 0 & 0 \\
\hline 70 & 3 & 1 & 360 & 15,42857143 & 3,888888889 \\
\hline 70 & 2,54 & 2 & 360 & 13,06285714 & 4,593175853 \\
\hline 70 & 1,38 & 3 & 360 & 7,097142857 & 8,45410628 \\
\hline 70 & 1,12 & 4 & 360 & 5,76 & 10,41666667 \\
\hline 70 & 0,89 & 5 & 360 & 4,577142857 & 13,10861423 \\
\hline 70 & 0,63 & 6 & 360 & 3,24 & 18,51851852 \\
\hline 70 & 0,4 & 7 & 360 & 2,057142857 & 29,16666667 \\
\hline 70 & 0,33 & 8 & 360 & 1,697142857 & 35,35353535 \\
\hline 70 & 0,21 & 9 & 360 & 1,08 & 55,55555556 \\
\hline
\end{tabular}

Tabla 1. Resultados obtenidos para la variación de la posición angular en función del voltaje 2010

Como se evidencia en la tabla anterior, la posición angular es directamente proporcional al voltaje aplicado en el actuador, por tal motivo, es necesario realizar la gráfica para determinar si los datos obtenidos teóricamente concuerdan con los datos experimentales como se observa en la figura 7.

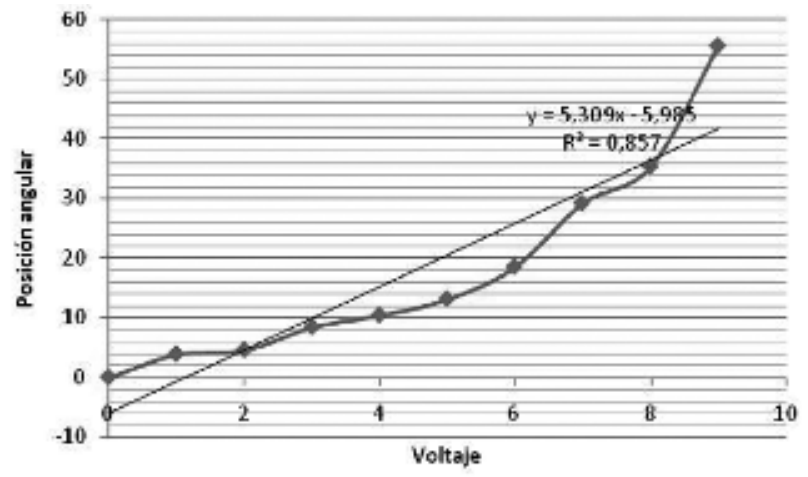

Figura 7. Gráfica de variación para la posición angular 2010

Finalmente, como se denota en la gráfica anterior, los resultados experimentales se asemejan a los resultados teóricos, pero fue necesaria la realización de una liberalización logarítmica debido a los posibles errores de medición e instrumentación, dicha linealización se aprecia como la línea más delgada en la gráfica anterior.

\section{Conclusiones}

- El modelo matemático del sistema del eslabón superior del robot, permite identificar la variación de la posición del mismo cuando aplica un voltaje sobre el motor a través de la función de transferencia del sistema.

- Las herramientas de software MatLAB ${ }^{\circledR}$ y Simulink ${ }^{\circledR}$, permiten analizar el sistema de una forma más sencilla, a través de la función de transferencia del sistema, ya sea por medio del código o del diagrama de bloques.

- Las respectivas simulaciones del sistema, permiten evidenciar la variación de la posición angular de la articulación, conociendo valores máximos y mínimos, que evidencien un desarrollo óptimo de las prácticas realizadas por los estudiantes.

\section{Referencias}

[1] Evans, D. (2004), "New approach to teaching robotics", disponible en: http://citeseerx.ist.psu.edu/se arch?q=Intelligent+tutors\&submit=Search\&sort=rlv, recuperado: 14 de diciembre de 2010

[2] Grega, W. \& Pilat, A. (2008), Real-Time control teaching using LEGO ${ }^{\circledR}$ MINDSTROMS ${ }^{\circledR}$ NXT robot, en Computer Science and Information Technology, 2008. IMCSIT 2008. International Multiconference on, pp. 625-628.

[3] Hirst, A. J.; Johnson, J., Petre, M.; Price, B. \& Richards, M. (2003), What is the best programming environment/language for teaching robotics using Lego Mindstorms?, en Artificial Life and Robotics, Volume 7, Number 3, pp. 124-131

[5] Joyce, B. \& Weil, M. (2002), Modelos de enseñanza,Gedisa.

[6] Kuo, B. (1996), Sistemas de Control Automático, séptima edición, Naucalpan de Juarez, Prentice Hall. [8] Lin, Chun-Hung; Liu, Eric Zhi-Feng; Kou, ChanHsin; Virnes, Marjo; Sutinen, Erkki \& Cheng, Shan-Shan (2009), A Case Analysis of Creative Spiral Instruction Model and Students' Creative Problem Solving Performance in a LEGO ${ }^{\circledR}$ Robotics Course, en Lectures Notes in Computer Science, Volume 5670/2009, pp. 501-505.

[7] Mosley, P. \& Doswell, J. (2008), The Virtual Instructor Intervention: A Case in LEGO Robotics, The International Journal of Virtual Reality , 7(1), pp. 15-20.

[9] Moundridou, M., \& Kalinoglou, A. (2008). Using LEGO Mindstorms as an Instructional Aid in Technical and Vocational Secondary Education: Experiences from an Empirical Case Study., en Lectures Notes in Computer Science, Volume 5192/2008, pp. 312-321.312,321. 
[4] Nourbakhsh, I.; Hamner, E.; Crowley, K. \& Wilkinson, K. (2004), Formal measures of learning in a secondary school mobile robotics course, en Robotics and Automation, 2004. Proceedings. ICRA '04. 2004 IEEE International Conference on

[10] O'Hara, K. \& Kay, J. (2005). "Investigating Open Source Software and Educational Robotics", disponible en: http://citeseer.ist.psu.edu/, recuperado: 10 de febrero de 2010

Diana J. Lancheros Cuesta. Ingeniera de Diseño y Automatización Electrónica, Especialista en multimedia para la docencia, Magíster en Tecnologías de la Información Aplicadas a la Educación, Estudiante de doctorado en Ingeniería de la Pontificia Universidad Javeriana de Bogotá Colombia. Su experiencia de 12 años ha sido llevada a cabo en gestión académica, docencia e investigación en diseño, desarrollo, implementación de sistemas automatizados, software, aplicaciones en Internet, y lo concerniente a las áreas de mecánica, sistemas y electrónica; Diseño e implementación de proyectos de investigación en áreas de tecnología para discapacitados, ambientes virtuales de aprendizaje, simulación para la predicción y análisis de comportamientos en sistemas sociales, electrónicos y de información.dlancheros@gmail.com 